\title{
Comparative efficacy and safety of tyrosine kinase inhibitors for thyroid cancer: a systematic review and meta-analysis
}

\author{
Takaaki Oba, Tatsunori Chino, Ai Soma, Tadafumi Shimizu, Mayu Ono, Tokiko Ito, Toshiharu Kanai, \\ Kazuma Maeno and Ken-ichi Ito
}

Division of Breast and Endocrine Surgery, Department of Surgery, Shinshu University School of Medicine, Matsumoto 390-8621, Japan

\begin{abstract}
The tyrosine kinase inhibitors (TKIs) sorafenib, lenvatinib, vandetanib, and cabozantinib are currently used for thyroid cancer treatment; however, the differences in their clinical efficacy and toxicity remain unclear. This meta-analysis assessed the efficacy and toxicity of these four TKIs based on 34 studies. The pooled incidence of partial response (PR), stable disease (SD), TKI-related adverse events (AEs), and pooled median progression-free survival (PFS) were calculated with $95 \%$ confidence intervals (CI). Complete response to TKIs was extremely rare $(0.3 \%)$. The highest PR rate and longest PFS were observed for lenvatinib in differentiated thyroid cancer (69\%, 95\% CI: 57-81 and 19 months, 95\% CI: 9-29, respectively) and vandetanib in medullary thyroid cancer (40\%, 95\% CI: $25-56$ and 31 months, $95 \%$ CI: 19-43, respectively). Although the discontinuation rate due to AEs was similar for each TKI, there was a difference in the most frequently observed AE for each TKI (hand-foot syndrome for sorafenib, hypertension and proteinuria for lenvatinib, and QTc prolongation for vandetanib). The identified differences in the TKI efficacy and AE profiles may provide a better understanding of thyroid cancer treatment. Although TKIs are promising agents for thyroid cancer treatment, they are unlikely to lead to a cure. Thus, even in the TKI era, a multimodal treatment including surgery, radioiodine therapy, external beam radiotherapy, and TKIs is required to optimize patient chances of improved survival.
\end{abstract}

Key words: Thyroid cancer, Tyrosine kinase inhibitor, Adverse event, Meta-analysis

THYROID CANCER is the most prevalent endocrine malignancy, which accounts for approximately $4 \%$ of all malignancies. Its overall incidence has increased over the years $[1,2]$. Differentiated thyroid carcinoma (DTC) is the most prevalent type of thyroid cancer, accounting for $90 \%-95 \%$ of all cases [2]. Although DTC is generally an indolent tumor, distant metastases may occasionally develop [3]. Radioiodine (RAI) therapy is beneficial for the control of metastatic DTC and can lead to tumor remission [4]. However, some metastatic lesions show primary or acquired resistance to RAI therapy. Since the therapeutic options for patients with RAI-refractory DTC (RR-DTC) are limited, a subset of these patients succumb to the disease; thus, the reported 10-year survival rate of patients with RR-DTC was 70\% [5]. Anaplastic thyroid carcinoma (ATC) accounts for less than $2 \%$ of all

Submitted Mar. 25, 2020; Accepted Jul. 13, 2020 as EJ20-0171 Released online in J-STAGE as advance publication Aug. 18, 2020 Correspondence to: Ken-ichi Ito, Division of Breast and Endocrine Surgery, Department of Surgery, Shinshu University School of Medicine, 3-1-1 Asahi, Matsumoto, Nagano 390-8621, Japan. E-mail: kenito@shinshu-u.ac.jp thyroid carcinomas but presents with a very aggressive clinical course [6]. Although surgery has been the most effective treatment to reduce the tumor burden of ATC [7-9], the prognosis remains very poor with mean survival time after diagnosis of 6 months [10]. Furthermore, the survival rate of patients with ATC has not improved over the past 20 years [9]. Medullary thyroid carcinoma (MTC) is also an uncommon type of thyroid cancer, a subset of which is a hereditary form associated with multiple endocrine neoplasia type 2A (MEN 2A), MEN 2B, and familial MTC [11, 12]. Ito et al. demonstrated that the prognosis of MTC patients was generally good: the 10- and 20-year cause-specific survival rates were $97.0 \%$ and $94.3 \%$, respectively [13]. RAI therapy cannot be applied to MTC, because MTC cells, which originate from thyroid $\mathrm{C}$ cells, do not take iodine up. Therefore, if distant metastases develop, the therapeutic options for MTC are limited. The described limited treatment choices for each histological type of thyroid cancer have aroused the need for a novel therapeutic strategy, and tyrosine kinase inhibitors (TKIs) have emerged as promising drugs in the last decade. 
Since positive results were published from two phase III clinical trials (the DECISION and SELECT trials) $[14,15]$, sorafenib and lenvatinib have been approved and are now available for the treatment of RR-DTC. As for MTC, two TKIs - vandetanib and cabozantinibhave been approved based on two phase III clinical trials (the ZETA and EXAM trials) [16, 17]. Sorafenib and lenvatinib have also been tested in phase II clinical trials and shown to be an effective treatment for advanced MTC [18-23]. Regarding ATC, lenvatinib has shown the potential to improve ATC tumor control and prognosis [23] and has thus been approved for the treatment of ATC in Japan.

Multimodal therapeutic approaches, including surgery, RAI therapy, external beam radiotherapy, and TKIs, will be further developed in the next decade. Specifically, given the increasing evidence of promising results with TKIs in clinical trials, TKIs may play an important role in thyroid cancer treatment. Even though there are currently four TKIs available for the treatment of thyroid cancer, the differences in their clinical efficacy and toxicity are still unclear. Therefore, in this study, we aimed to systematically review the existing literature and meta-analyze the efficacy and safety of the four TKIs (sorafenib, lenvatinib, cabozantinib, and vandetanib) to elucidate the differences among these drugs for the treatment of thyroid cancer.

\section{Materials and Methods}

\section{Search strategies}

We searched the databases PubMed, Scopus, and ClinicalTrials.gov for relevant studies published up to July 2019 using the search terms "thyroid AND (cancer OR carcinoma) AND (sorafenib, OR lenvatinib, OR vandetanib, OR cabozantinib)". We performed the analysis according to the Preferred Reporting Items for Systemic Reviews and Meta-Analyses (PRISMA) statement [24]. All studies were assessed individually by two of the authors (TO and MO).

\section{Selection criteria}

We selected studies, which fulfilled the following inclusion criteria: (a) clinical trials or observational longitudinal studies, in which the response rate and adverse events (AEs) were reported according to the Response Evaluation Criteria in Solid Tumors (RECIST) and Common Terminology Criteria for Adverse Events (CTCAE), respectively; (b) except for ATC, sorafenib, lenvatinib, vandetanib, and cabozantinib were administered at initial doses of $400 \mathrm{mg}$ twice a day, $24 \mathrm{mg}$ a day, $300 \mathrm{mg}$ a day, and $140 \mathrm{mg}$ a day, respectively; (c) patients were over 18 years of age; and (d) the articles were written in English.
Exclusion criteria were: (a) studies, in which TKIs were combined with other treatments; (b) case reports; (c) literature reviews; and (d) duplicate studies.

\section{Data extraction}

The following data were extracted: author name, country, year of publication, study design, and enrolled patient characteristics. Enrolled patient characteristics included age; sex; the Eastern Cooperative Oncology Group performance status; histological type of thyroid cancer; pretreatment; initial dose; response rate [complete response (CR), partial response (PR), and stable disease (SD)]; progression-free survival (PFS); and overall survival (OS). For AEs, we extracted data only from prospective studies, considering the possibility that AEs were overlooked in retrospective observational longitudinal studies (RSs).

\section{Statistical analysis}

We performed this meta-analysis using JAMOVI, a software built on the $\mathrm{R}$ statistical language. The overall pooled rates for $\mathrm{PR}, \mathrm{SD}, \mathrm{AEs}$, pooled median PFS, and pooled median OS were calculated with the corresponding $95 \%$ confidence intervals (CI) for each drug. The overall pooled discontinuation rate was also evaluated. Heterogeneity was assessed through $I^{2}$ statistics. $I^{2}>$ $50 \%$ and $I^{2} \leq 50 \%$ were classified as high and low heterogeneity, respectively. Egger's test was carried out to evaluate the publication bias, and a $p$ value $<0.05$ was considered statistically significant publication bias.

\section{Results}

\section{Study selection}

A total of 1,152 potentially relevant articles were identified. Among them, 1,111 articles were eliminated after reading their titles and abstracts. After reading the full texts of the remaining 41 articles, seven articles were excluded due to treatment with a reduced starting dose or inability to extract data for the meta-analysis. Finally, a total of 34 studies with 1,713 patients (14 studies for sorafenib [15, 18-21, 25-33]; 13 studies for lenvatinib $[14,22,23,34-43]$; five studies for vandetanib [17, 44-47]; and two studies for cabozantinib [48, 49]) were included in this systematic review. Details on the literature search are presented in Supplementary Fig. 1.

The characteristics of the included 34 articles are summarized in Table 1. With regards to sorafenib, eight studies were phase II or III clinical trials [15, 18-21, 25, $29,30]$, one study was a prospective observational longitudinal study (PS) [33], and the remaining five studies were RSs [26-28, 31, 32]. Totally, 523 patients (DTC: $n$ $=381$, MTC: $n=103$, and ATC: $n=39$ ) were enrolled in 
Table 1 Descriptive summary of the inclusion studies

\begin{tabular}{|c|c|c|c|c|c|c|c|c|c|}
\hline Sorafenib & Study type & Countries & Patients & DTC & MTC & ATC & Age & Prior TKIs & ECOG PS \\
\hline Brose et al. ${ }^{[15]}$ & Phase III & Multi country & 207 & 207 & 0 & 0 & 63 & - & $0-2$ \\
\hline Ahmed et al. ${ }^{[18]}$ & Phase II & UK & 34 & 19 & 15 & 0 & 55 & - & $0-1$ \\
\hline Gupta et al. ${ }^{[19]}$ & Phase II & US & 30 & 27 & 1 & 2 & 63 & - & $0-1$ \\
\hline Kloos et al. ${ }^{[25]}$ & Phase II & US & 56 & 52 & 0 & 4 & N.D & - & N.D \\
\hline Sneider et al. ${ }^{[29]}$ & Phase II & Netherlands & 31 & 31 & 0 & 0 & 64 & - & $0-2$ \\
\hline Cabanillas et al. ${ }^{[26]}$ & RS & US & 15 & 15 & 0 & 0 & 61 & - & N.D \\
\hline Ito et al..$^{[20]}$ & Phase II & Japan & 18 & 0 & 8 & 10 & 64 & - & $0-1$ \\
\hline Capdevila et al. ${ }^{[28]}$ & RS & Spain & 34 & 16 & 15 & 3 & 57.3 & - & $0-2$ \\
\hline Benekli et al. ${ }^{[31]}$ & RS & Turkey & 30 & 14 & 16 & 0 & 57 & - & $0-2$ \\
\hline Lam et al. ${ }^{[21]}$ & Phase II & US & 21 & 0 & 21 & 0 & 60 & - & $0-2$ \\
\hline Frank-Raue et al. ${ }^{[27]}$ & RS & Germany & 5 & 0 & 5 & 0 & 50 & + & N.D \\
\hline Koscic et al. ${ }^{[33]}$ & PS & Hungary & 10 & 0 & 10 & 0 & 51.7 & - & $0-1$ \\
\hline de Castroneves et al. ${ }^{[32]}$ & RS & Brazil & 12 & 0 & 12 & 0 & 48 & + & N.D \\
\hline Savvides et al. ${ }^{[30]}$ & Phase II & US & 20 & 0 & 0 & 20 & 59 & - & $0-2$ \\
\hline Total & & & 523 & 381 & 103 & 39 & & & \\
\hline Lenvatinib & Study type & Countries & Patients & DTC & MTC & ATC & Age & Prior TKIs & ECOG PS \\
\hline Schlumberger et al $^{[14]}$ & Phase III & Multi country & 261 & 261 & 0 & 0 & 64 & + & $0-3$ \\
\hline Cabanillas et al..$^{[35]}$ & Phase II & Multi country & 58 & 58 & 0 & 0 & 63 & + & $0-2$ \\
\hline Takahashi et al..$^{[23]}$ & Phase II & Japan & 51 & 25 & 9 & 17 & 61 & + & $0-2$ \\
\hline Yamazaki et al..$^{[43]}$ & RS & Japan & 30 & 30 & 0 & 0 & 68 & + & N.D \\
\hline Suzuki et al. ${ }^{[42]}$ & RS & Japan & 26 & 26 & 0 & 0 & 64 & + & $0-3$ \\
\hline Balmelli et al. ${ }^{[34]}$ & RS & Switzerland & 13 & 13 & 0 & 0 & 72 & + & $0-2$ \\
\hline Nervo et al. ${ }^{[40]}$ & RS & Italy & 12 & 12 & 0 & 0 & 61.1 & + & $0-2$ \\
\hline Schlumberger et al. ${ }^{[22]}$ & Phase II & Multi country & 59 & 0 & 59 & 0 & 51.6 & + & $0-2$ \\
\hline Iwasaki et al..$^{[37]}$ & RS & Japan & 23 & 0 & 0 & 23 & 77 & - & $0-2$ \\
\hline lyer et al. ${ }^{[38]}$ & RS & US & 10 & 0 & 0 & 10 & N.D & - & N.D \\
\hline Koyama et al. ${ }^{[39]}$ & $\mathrm{RS}$ & Japan & 5 & 0 & 0 & 5 & 58 & - & 0 \\
\hline Staub et al..$^{[41]}$ & RS & Japan & 3 & 0 & 0 & 3 & 66 & + & $0-3$ \\
\hline Iniguez-Ariza et al. ${ }^{[36]}$ & RS & US & 3 & 0 & 0 & 3 & 67 & - & $2-3$ \\
\hline Total & & & 554 & 425 & 68 & 61 & & & \\
\hline
\end{tabular}

\begin{tabular}{|c|c|c|c|c|c|c|c|c|c|}
\hline Vandetanib & Study type & Countries & Patients & DTC & MTC & ATC & Age & Prior TKIs & ECOG PS \\
\hline Leboullex et al. ${ }^{[45]}$ & Phase II & Multi country & 72 & 72 & 0 & 0 & 63 & + & $0-2$ \\
\hline Wells et al. ${ }^{[17]}$ & Phase III & Multi country & 231 & 0 & 231 & 0 & 50.7 & - & $0-2$ \\
\hline Hu et al..$^{[47]}$ & Phase IV & Multi country & 41 & 0 & 41 & 0 & 52.7 & + & $0-2$ \\
\hline Wells et al..$^{[44]}$ & Phase II & Multi country & 30 & 0 & 30 & 0 & 49 & + & $0-2$ \\
\hline Werner et al..$^{[46]}$ & RS & Germany & 18 & 0 & 18 & 0 & 46.5 & + & N.D \\
\hline Total & & & 392 & 72 & 320 & 0 & & & \\
\hline Cabozantinib & Study type & Countries & Patients & DTC & MTC & ATC & Age & Prior TKIs & ECOG PS \\
\hline Cabanillas et al. ${ }^{[49]}$ & Phase II & US & 25 & 25 & 0 & 0 & 64 & + & $0-1$ \\
\hline Elisei et al. ${ }^{[48]}$ & Phase III & Multi country & 219 & 0 & 219 & 0 & 55 & + & $0-2$ \\
\hline Total & & & 244 & 25 & 219 & 0 & & & \\
\hline
\end{tabular}

RS, retrospective study; PS, prospective study; DTC, differentiated thyroid carcinoma; MTC, medullary thyroid carcinoma; ATC, anaplastic thyroid carcinoma; TKI, tyrosine kinase inhibitor; ECOG, Eastern Cooperative Oncology Group; PS, performance status; N.D, not described 
this meta-analysis. Studies on lenvatinib included four phase II or III clinical trials [14, 22, 23, 35] and nine RSs [34, 36-43], comprising totally 554 patients (DTC: $n=$ 425, MTC: $n=68$, and ATC: $n=61$ ). For vandetanib and cabozantinib, all studies except one [46] were clinical trials (Phase II, III, or IV) [17, 44, 45, 47-49], in which 392 (DTC: $n=72$ and MTC: $n=320$ ) and 244 patients (DTC: $n=25$ and MTC: $n=219$ ) were treated, respectively. We could not conduct a meta-analysis for cabozantinib due to the low number of included studies.

\section{Efficacy}

Overall, CR was reported with a remarkably low incidence ( 5 out of 1,713 patients; $0.3 \%$ ). Among the five patients who showed CR, four were DTC patients treated with lenvatinib [14], whereas one was an MTC patient treated with vandetanib [47].

Next, we meta-analyzed the PR rate, SD rate, and PFS for each pathological type of thyroid cancer (DTC, MTC, and ATC) (Table 2). The meta-analysis for OS could be performed only for ATC, because OS data were lacked in most studies of DTC and MTC.

DTC

In terms of $\mathrm{PR}$, lenvatinib was superior to sorafenib: $15 \%$ (95\% CI: $\left.5-26, I^{2}=0 \%, p=0.48\right)$ for sorafenib and $69 \%$ (95\% CI: $57-81, I^{2}=15.7 \%, p=0.29$ ) for lenvatinib. Contrarily, the pooled SD rate was lower with lenvatinib than with sorafenib treatment: 59\% (95\% CI: $37-81, I^{2}=65.2 \%, p=0.86$ ) for sorafenib and $27 \%$ (95\% CI: $11-43, I^{2}=36.2 \%, p=0.62$ ) for lenvatinib. The low SD rate with lenvatinib treatment may have been induced by the high $\mathrm{PR}$ rate. Consistent with the higher PR rate for lenvatinib treatment, the pooled PFS was longer for lenvatinib than for sorafenib: 14 months (95\% CI: 3-24, $I^{2}=0 \%, p=0.66$ ) for sorafenib and 19 months (95\% CI: 9-29, $\left.I^{2}=0 \%, p=0.95\right)$ for lenvatinib.

No meta-analysis could be performed for vandetanib and cabozantinib due to the small number of articles. However, the findings of two phase II trials showed that the median PFS was 11.1 months (95\% CI: 7.7-14.0) for vandetanib and 12.7 months (95\% CI: 10.9-34.7) for cabozantinib [45, 49], which was inferior to the pooled PFS for lenvatinib.

MTC

The pooled PR and SD rates for sorafenib treatment were $23 \%$ (95\% CI: $\left.1-45, I^{2}=0 \%, p=0.61\right)$ and $77 \%$ (95\% CI: 45-100, $I^{2}=48.9 \%, p=0.42$ ), respectively, whereas those for vandetanib treatment were $40 \%(95 \%$ CI: $\left.25-56, I^{2}=21.0 \%, p=0.21\right)$ and $59 \%$ (95\% CI: $37-$ $81, I^{2}=51.6 \%, p=0.32$ ), respectively. The pooled PFS for vandetanib was longer than that for sorafenib treatment: 16 months (95\% CI: $\left.0-45, I^{2}=0 \%, p=0.94\right)$ for sorafenib and 31 months (95\% CI: 19-43, $I^{2}=0 \%, p=$ 0.81 ) for vandetanib.

No meta-analysis could be performed for lenvatinib and cabozantinib due to the limited number of eligible studies. Lenvatinib was tested in a phase II study and showed 9.0 months (95\% CI: 7.0-not evaluable) PFS for MTC [22]. Regarding cabozantinib, the median PFS in a phase III trial (EXAM trial) was significantly longer in the cabozantinib arm (11.2 months) than in the placebo arm (4.0 months) (hazard ratio $0.28,95 \%$ CI $0.19-0.40$,

Table 2 Pooled incidence of partial response, stable disease, median progression-free survival, and median overall survival

\begin{tabular}{|c|c|c|c|c|c|c|c|c|c|c|c|c|}
\hline & Sorafenib & $95 \% \mathrm{CI}$ & $r^{2}$ & $p$ & Lenvatinib & $95 \% \mathrm{CI}$ & $r^{2}$ & $p$ & Vandetanib & $95 \% \mathrm{CI}$ & $r^{2}$ & $p$ \\
\hline \multicolumn{13}{|l|}{ DTC } \\
\hline PR & $15 \%$ & $5-26$ & $0 \%$ & 0.48 & $69 \%$ & $57-81$ & $15.7 \%$ & 0.29 & & & & \\
\hline SD & $59 \%$ & $37-81$ & $65.2 \%$ & 0.86 & $27 \%$ & $11-43$ & $36.2 \%$ & 0.62 & & & & \\
\hline PFS & 14 months & $3-24$ & $0 \%$ & 0.66 & 19 months & $9-29$ & $0 \%$ & 0.95 & & & & \\
\hline \multicolumn{13}{|l|}{ MTC } \\
\hline PR & $23 \%$ & $1-45$ & $0 \%$ & 0.61 & & & & & $40 \%$ & $25-56$ & $21.0 \%$ & 0.21 \\
\hline SD & $77 \%$ & $45-100$ & $48.9 \%$ & 0.42 & & & & & $59 \%$ & $37-81$ & $51.6 \%$ & 0.32 \\
\hline PFS & 16 months & $0-45$ & $0 \%$ & 0.94 & & & & & 31 months & $19-43$ & $0 \%$ & 0.81 \\
\hline \multicolumn{13}{|l|}{ ATC } \\
\hline PR & & & & & $24 \%$ & $0-54$ & $0 \%$ & 0.46 & & & & \\
\hline SD & & & & & $50 \%$ & $14-87$ & $25.0 \%$ & 0.98 & & & & \\
\hline PFS & & & & & 5 months & $1-9$ & $25.0 \%$ & 0.98 & & & & \\
\hline OS & & & & & 11 months & $0-22$ & $91.4 \%$ & 0.65 & & & & \\
\hline
\end{tabular}

DTC, differentiated thyroid carcinoma; MTC, medullary thyroid carcinoma; ATC, anaplastic thyroid carcinoma; PR, partial response; SD, stable disease; PFS, progression-free survival; OS, overall survival; $\mathrm{CI}$, confidence interval; $p, p$ value identified by Egger's regression test 
$p<0.01$ ) [48]. These reported PFS for lenvatinib and cabozantinib was shorter than the pooled PFS for vandetanib.

ATC

We could perform a meta-analysis only for lenvatinib treatment. The pooled PR and SD rates were 24\% (95\% CI: $\left.0-54, I^{2}=0 \%, p=0.46\right)$ and $50 \%\left(95 \% \mathrm{CI}: 14-87, I^{2}\right.$ $=25.0 \%, p=0.98)$. The pooled PFS was 5 months $(95 \%$ CI: $\left.1-9, I^{2}=25.0 \%, p=0.98\right)$. OS data were available in five articles [23, 36-39], and the pooled OS was 11 months (95\% CI: $\left.0-22, I^{2}=91.4 \%, p=0.65\right)$. Although we could not conduct a meta-analysis for sorafenib treatment due to the lack of eligible articles, the reported PFS and OS for ATC were 1.9-4.4 months and 3.9-5.0 months, respectively [20, 28, 30].

\section{Adverse events}

Data on dose reduction or drug interruption or discontinuation due to AEs were obtained from 12 [15, 18-21, 25, 27-29, 31-33], eight [14, 22, 23, 34, 35, 40, 42, 43], and four studies [17, 44, 45, 47] for sorafenib, lenvatinib, and vandetanib, respectively. The dose reduction and drug interruption rates were highest for lenvatinib (Supplementary Table 1). However, the pooled discontinuation rate was equivalent for the three TKIs (sorafenib: 16\%, 95\% CI: 6-26, $I^{2}=0 \%, p=0.62$; lenvatinib: $16 \%$, 95\% CI: 7-25, $I^{2}=0 \%, p=0.71$; and vandetanib: $19 \%$, 95\% CI: 6-31, $I^{2}=18.2 \%, p=0.57$ ) (Table 3).

The AEs associated with each TKI and their pooled rates are summarized in Table 4. Diarrhea was a frequent $\mathrm{AE}$ in sorafenib, lenvatinib, and vandetanib treatment. With sorafenib treatment, the incidence of skin-related AEs such as hand-foot syndrome (HFS) and rash was extremely high (HFS, all grades: 96\%, 95\% CI: 86-100, $I^{2}=0.4 \%, p=0.49$; Grade 3 or $4: 21 \%, 95 \%$ CI: $11-31$, $I^{2}=0 \%, p=0.99$; rash, all grades: $79 \%, 95 \% \mathrm{CI}: 54$ $100, I^{2}=77.2 \%, p=0.56$; and Grade 3 or $4: 6 \%, 95 \%$ CI: $\left.0-16, I^{2}=0 \%, p=0.89\right)$. Skin-related AEs were not as common with lenvatinib as with sorafenib treatment, but hypertension was reported with a remarkably high incidence (all grades: 96\%, 95\% CI: 59-100, $I^{2}=90.9 \%$, $p=0.59$ and Grade 3 or $4: 28 \%, 95 \%$ CI: $8-49, I^{2}=$ $71.1 \%, p=0.39$ ). In addition, proteinuria was documen-

Table 3 Pooled discontinuation rate of TKI treatment

\begin{tabular}{lcccc}
\hline & Discontinuation rate & $95 \% \mathrm{CI}$ & $I^{2}$ & $p$ \\
\hline Sorafenib & $16 \%$ & $6-26$ & $0 \%$ & 0.62 \\
Lenvatinib & $16 \%$ & $7-25$ & $0 \%$ & 0.71 \\
Vandetanib & $19 \%$ & $6-31$ & $18.2 \%$ & 0.57 \\
\hline
\end{tabular}

TKI, tyrosine kinase inhibitor; CI, confidence interval; $p, p$ value identified by Egger's regression test ted with higher incidence for lenvatinib than sorafenib and vandetanib treatment (lenvatinib, all grades: $45 \%$, 95\% CI: 9-80, $I^{2}=90.9 \%, p=0.33$ and Grade 3 or 4 : $3 \%, 95 \% \mathrm{CI}: 0-13, I^{2}=0 \%, p=0.33$; and sorafenib and vandetanib, not applicable for a meta-analysis due to the lack of data). Vandetanib treatment was associated with higher incidence of QTc prolongation than sorafenib and lenvatinib treatment (sorafenib and lenvatinib, not applicable for a meta-analysis due to the lack of data; vandetanib, all grades: $19 \%, 95 \% \mathrm{CI}: 8-29, I^{2}=0 \%, p=0.19$; and Grade 3 or $4: 10 \%, 95 \%$ CI: $0-21, I^{2}=0 \%, p=$ 0.48).

Although we could not conduct a meta-analysis on the reasons for dose reduction or drug interruption or discontinuation due to the lack of available studies, diarrhea was reported as one of the major reasons in phase II or III clinical trials for lenvatinib and vandetanib treatment [14, 44, 45]. Other main causes included hypertension and proteinuria for lenvatinib [14] and QTc prolongation for vandetanib [45]. The most frequently reported $\mathrm{AE}$, which lead to a dose reduction of the sorafenib treatment, was HFS [15, 18-21]. The timing of the dose reduction was within 2 months, 2.2-3.0 months, and 4.9 months for sorafenib [18, 21, 29], lenvatinib [14, 22], and vandetanib [44], respectively.

TKI treatment was associated with fatal AEs such as tumor-related bleeding in various cancer types [50]. Indeed, fatal AEs were also documented with thyroid cancer treatment, although they were rare events $[28,35$, 45]. The total incidence of deaths caused by fatal AEs associated with each TKI was $0.7 \%(n=4$ out of 523 patients), $1.9 \%$ ( $n=11$ out of 554 patients), and $1.7 \%$ ( $n$ $=7$ out of 392 patients) for the treatment with sorafenib, lenvatinib, and vandetanib, respectively. Particularly, tumor-related arterial hemorrhage was documented as a perilous fatal $\mathrm{AE}$ associated with lenvatinib treatment [35].

\section{Discussion}

In this meta-analysis, several clinically important findings were identified: (1) Regarding the PR rate and PFS, lenvatinib was superior to sorafenib for DTC treatment. (2) Vandetanib treatment showed the most favorable PFS for MTC treatment. (3) The prognosis of ATC remained very poor even with TKI treatment. (4) CR was extremely rare with TKI treatment. (5) Drug discontinuation due to AEs occurred with equivalent incidence for each TKI in approximately $16 \%-19 \%$ of the treated patients. (6) Each TKI was characterized by specific AEs with high incidence (skin-related AEs, hypertension and proteinuria, and QTc prolongation for sorafenib, lenvatinib, and vandetanib, respectively). 
Table 4 Adverse events associated with sorafenib, lenvatinib, and vandetanib treatment

\begin{tabular}{|c|c|c|c|c|c|c|c|c|c|c|c|c|}
\hline \multirow{2}{*}{$\begin{array}{l}\text { Adverse events } \\
\text { All grades }\end{array}$} & \multicolumn{4}{|c|}{ Sorafenib } & \multicolumn{4}{|c|}{ Lenvatinib } & \multicolumn{4}{|c|}{ Vandetanib } \\
\hline & $\begin{array}{l}\text { Pooled } \\
\text { frequency }\end{array}$ & $95 \% \mathrm{CI}$ & $I^{2}$ & $p$ & $\begin{array}{l}\text { Pooled } \\
\text { frequency }\end{array}$ & $95 \% \mathrm{CI}$ & $I^{2}$ & $p$ & $\begin{array}{l}\text { Pooled } \\
\text { frequency }\end{array}$ & $95 \% \mathrm{CI}$ & $I^{2}$ & $p$ \\
\hline HFS & $96 \%$ & $86-100$ & $0.4 \%$ & 0.49 & $45 \%$ & $9-80$ & $90.1 \%$ & 0.61 & N.A & N.A & N.A & N.A \\
\hline Hypertension & $41 \%$ & $31-51$ & $0.0 \%$ & 0.51 & $96 \%$ & 59-100 & $90.9 \%$ & 0.59 & $36 \%$ & $26-46$ & $0 \%$ & 0.66 \\
\hline Proteinuria & N.A & N.A & N.A & N.A & $45 \%$ & $9-80$ & $90.9 \%$ & 0.33 & N.A & N.A & N.A & N.A \\
\hline Mucositis & $30 \%$ & $19-40$ & $3.1 \%$ & 0.09 & $38 \%$ & $24-51$ & $35.5 \%$ & 0.82 & N.A & N.A & N.A & N.A \\
\hline Diarrhea & $81 \%$ & $69-92$ & $12.5 \%$ & 0.08 & $75 \%$ & $62-88$ & $33.5 \%$ & 0.55 & $73 \%$ & $52-95$ & $67 \%$ & 0.87 \\
\hline Fatigue & $62 \%$ & $41-84$ & $67.1 \%$ & 0.42 & $70 \%$ & $60-79$ & $0.01 \%$ & 0.63 & $31 \%$ & $12-50$ & $57.2 \%$ & 0.28 \\
\hline Weight loss & $59 \%$ & $38-79$ & $67.1 \%$ & 0.30 & $53 \%$ & $33-73$ & $67.6 \%$ & 0.96 & N.A & N.A & N.A & N.A \\
\hline Rash & $79 \%$ & $54-100$ & $77.2 \%$ & 0.56 & $14 \%$ & $4-24$ & $0 \%$ & 0.44 & $44 \%$ & $26-63$ & $57.1 \%$ & 0.59 \\
\hline Anorexia & $35 \%$ & $17-53$ & $39.2 \%$ & 0.46 & $67 \%$ & $44-90$ & $76.2 \%$ & 0.45 & $24 \%$ & $14-34$ & $0 \%$ & 0.43 \\
\hline Alopecia & $71 \%$ & $55-87$ & $24.1 \%$ & 0.21 & $13 \%$ & $3-23$ & $0 \%$ & 0.57 & N.A & N.A & N.A & N.A \\
\hline $\begin{array}{l}\text { ECG QT } \\
\text { prolonged }\end{array}$ & N.A & N.A & N.A & N.A & N.A & N.A & N.A & N.A & $19 \%$ & $8-29$ & $0 \%$ & 0.19 \\
\hline \multicolumn{13}{|l|}{ Grade 3,4} \\
\hline HFS & $21 \%$ & $11-31$ & $0 \%$ & 0.99 & $3 \%$ & $0-13$ & $0 \%$ & 0.94 & N.A & N.A & N.A & N.A \\
\hline Hypertension & $9 \%$ & $0-19$ & $0 \%$ & 0.91 & $28 \%$ & $8-49$ & $71.1 \%$ & 0.39 & $8 \%$ & $0-18$ & $0 \%$ & 0.98 \\
\hline Proteinuria & N.A & N.A & N.A & N.A & $3 \%$ & $0-13$ & $0 \%$ & 0.33 & N.A & N.A & N.A & N.A \\
\hline Mucositis & $2 \%$ & $0-12$ & $0 \%$ & 0.86 & $3 \%$ & $0-13$ & $0 \%$ & 0.76 & N.A & N.A & N.A & N.A \\
\hline Diarrhea & $5 \%$ & $0-15$ & $0 \%$ & 0.84 & $9 \%$ & $0-19$ & $0 \%$ & 0.72 & $11 \%$ & $0-21$ & $0 \%$ & 0.94 \\
\hline Fatigue & $7 \%$ & $0-17$ & $0 \%$ & 0.82 & $9 \%$ & $0-19$ & $0 \%$ & 0.83 & $5 \%$ & $0-15$ & $0 \%$ & 0.89 \\
\hline Weight loss & $5 \%$ & $0-15$ & $0 \%$ & 0.83 & $9 \%$ & $0-19$ & $0 \%$ & 0.87 & N.A & N.A & N.A & N.A \\
\hline Rash & $6 \%$ & $0-16$ & $0 \%$ & 0.89 & $3 \%$ & $0-13$ & $0 \%$ & 0.72 & $3 \%$ & $0-14$ & $0 \%$ & 0.90 \\
\hline Anorexia & $2 \%$ & $0-13$ & $0 \%$ & 0.98 & $2 \%$ & $0-12$ & $0 \%$ & 0.49 & $3 \%$ & $0-14$ & $0 \%$ & 0.88 \\
\hline Alopecia & N.A & N.A & N.A & N.A & N.A & N.A & N.A & N.A & N.A & N.A & N.A & N.A \\
\hline $\begin{array}{l}\text { ECG QT } \\
\text { prolonged }\end{array}$ & N.A & N.A & N.A & N.A & N.A & N.A & N.A & N.A & $10 \%$ & $0-21$ & $0 \%$ & 0.48 \\
\hline
\end{tabular}

HFS, hand foot syndrome; ECG, electrocardiogram; CI, confidence interval; $p, p$ value identified by Egger's regression test; N.A, not applicable

Sorafenib and lenvatinib are currently available for RR-DTC treatment. However, no definitive evidence exists on which drug may lead to better patient outcomes, because no clinical trials have compared sorafenib and lenvatinib directly. The result of the current meta-analysis indicates that lenvatinib has higher potential to cause tumor shrinkage and prolong the PFS than sorafenib. Notably, as described in Table 1, all studies on lenvatinib treatment of DTC included patients previously treated with other TKIs (mainly sorafenib). However, no patients previously treated with other TKIs were included in any of the sorafenib studies, because sorafenib was the first drug approved for RR-DTC treatment. Despite the different inclusion criteria, the longer PFS with lenvatinib treatment indicates that lenvatinib has superior anti-tumor potency to sorafenib and may be effective even for sorafenib-resistant DTC. Notably, lenvatinib has a broader range of target molecules than sorafenib; specifically, lenvatinib blocks signaling via the vascular endothelial growth factor receptor (VEGFR) 1 and fibroblast growth factor FGF receptor (FGFR), whereas sorafenib does not; representative target molecules are listed in Table 5. Thus, even after acquired resistance to sorafenib emerges, lenvatinib may delay tumor growth by inhibiting other distinct receptors such as VEGFR1 and FGFR. Altogether, lenvatinib may be a favorable choice for the treatment of RR-DTC in terms of its anti-tumor effect.

However, lenvatinib treatment was associated with a higher rate of dose reduction and treatment interruption 
Table 5 Currently available TKIs and their representative target molecules

\begin{tabular}{lcccccccc}
\hline Drugs & VEGFR1 & VEGFR2 & VEGFR3 & c-KIT & RET & PDGFR & FGFR1-3 & Raf \\
\hline Sorafenib & - & + & + & + & + & + & - & + \\
Lenvatinib & + & + & + & + & + & + & & + \\
Vandetanib & - & + & - & + & + & - & - & - \\
Cabozantinib & - & + & - & + & + & - & - & -
\end{tabular}

TKI, tyrosine kinase inhibitor; VEGFR, vascular endothelial growth factor receptor; KIT, v-kit Hardy-Zuckerman 4 feline sarcoma viral oncogene; RET, rearranged during transfection receptor; PDGFR, platelet-derived growth factor receptor; FGFR, fibroblast growth factor receptor; Raf, v-raf murine sarcoma viral oncogene

due to AEs than sorafenib. Besides, the severity of AEs may differ with sorafenib and lenvatinib treatment. For instance, lenvatinib-induced drastic tumor shrinkage may cause tumor-related bleeding, which in certain cases may lead to sudden death [35]. In this regard, the tumor invasion to surrounding tissues or organs such as vessels, esophagus, skin, or trachea has been identified as a risk factor for lenvatinib-induced tumor-related bleeding [41]. Therefore, sorafenib may be a better choice for a patient with a tumor involving those tissues or organs. Another severe AE associated with lenvatinib is tumor fistula formation, including the formation of skin, tracheoesophageal, and gastrointestinal fistulas [14, 22, 37]. Such fistulas caused by lenvatinib remarkably diminish the patient quality of life and may require long-term drug interruption or discontinuation. In addition, lenvatinib treatment causes renal impairment in some patients despite appropriate drug interruption and supportive care $[51,52]$. In cases when lenvatinib is administrated before sorafenib, and lenvatinib-induced renal dysfunction cannot be recovered even after drug discontinuation, the patient may lose the chance to be treated by sorafenib. Considering these severe AEs associated with lenvatinib treatment, sorafenib treatment prior to lenvatinib may be a better treatment choice for some DTC patients. In this regard, a biomarker for the prediction of treatment response and severe AEs would facilitate the selection of the initial drug. However, no such predictive marker has been identified up to date, and thus it should be explored in the future. Furthermore, a clinical trial comparing the efficacy and safety of lenvatinib following sorafenib and vice versa may provide the solution to this clinical problem.

As for MTC, the median PFS in the cabozantinib phase III trial (EXAM trial) (11.2 months) was considerably shorter than the pooled median PFS for vandetanib (31 months). However, the EXAM trial included patients previously treated with other TKIs, whereas the ZETA trial [17] (a phase III trial that tested the efficacy of vandetanib for MTC) enrolled only TKI-naive patients. Furthermore, an important inclusion criterion for the
EXAM trial was the requirement for radiographic progression per the RECIST within the prior 14 months. Importantly, the PFS duration for the placebo group in the EXAM trial was also inferior to that in the ZETA trial (19.3 months in the ZETA trial and 4.0 months in the EXAM trial). Therefore, the longer median PFS duration with vandetanib treatment may be explained by the different levels of disease severity. The comparison of vandetanib and sofafenib in this meta-analysis indicates that vandetanib (31 months) shows longer pooled PFS than sorafenib (16 months). Furthermore, the pooled PFS for vandetanib was longer than the PFS for lenvatinib (9.0 months) reported in a phase II study [22]. Therefore, although we cannot draw a definitive conclusion due to the different inclusion criteria for each study, vandetanib may be the most preferred drug for MTC with regards to PFS.

The differences in the TKI AE profiles identified in this study will facilitate the choice of an appropriate TKI for each patient in the clinical practice. These differential profiles of the analyzed TKIs may arise from their different molecular targets (Table 5). VEGFR inhibition, which is the common target for the TKIs investigated in this study, induces hypertension [53]. In addition to VEGFR, lenvatinib inhibits the FGF signaling pathway, which controls vascular function and blood pressure [54], whereas the other TKIs do not. In contrast, Raf is the characteristic target of sorafenib. Raf inhibition by sorafenib induces mast cell degranulation and maturation, which are associated with various types of skin diseases [55]. Therefore, these differences in molecular targets may result in the characterized different highly observed AEs for sorafenib (skin-related) and lenvatinib (hypertension). Early intervention and appropriate management for these common AEs are important to avoid adverse outcomes. For HFS, patient education and proactive management to keep skin well hydrated, remove calluses regularly, and use pain medication are essential to keep sorafenib dose. In addition, topical corticosteroids are effective to prevent worsening [56]. Once hypertension (systolic blood pressure is $\geq 140 \mathrm{mmHg}$ or diastolic 
blood pressure is $>90 \mathrm{mmHg}$ ) has been induced by TKI treatment, antihypertensive drugs including calcium channel blockers, angiotensin-converting enzyme inhibitors, angiotensin II receptor blockers should be administrated. These drugs can be used as single therapy or in combination [57].

Given the high rate of dose reduction or drug interruption and the non-negligible drug discontinuation rate due to AEs, an optimal dose that is better tolerated but presents comparable efficacy to the full dose should be explored for each TKI. Indeed, the results of a phase IV study, which evaluated the benefit-risk of two starting doses $(150 \mathrm{mg} /$ day or $300 \mathrm{mg} /$ day) of vandetanib in patients with MTC, were published recently [47]. In this trial, the $300 \mathrm{mg}$ dose showed a more favorable overall response rate than the $150 \mathrm{mg}$ dose (150 mg: $20.0 \%$, 95\% CI: $10.5-34.8$ and $300 \mathrm{mg}: 29.3 \%$, 95\% CI: $17.6-$ 44.5). However, with regards to safety, a lower discontinuation rate due to AEs was observed with the $150 \mathrm{mg}$ dose (150 mg: $5.0 \%$ and $300 \mathrm{mg}$ : 12.2\%). These results indicate that the appropriate starting dose is $300 \mathrm{mg}$; however, a lower initial dose may reduce toxicity. Regarding lenvatinib, a post hoc analysis of the SELECT study revealed that patients with shorter dose interruptions showed longer PFS than those with longer interruptions [58]. These results highlight the importance of dose intensity maintenance with appropriate AE management. However, the remarkably high dose reduction and treatment interruption rate with lenvatinib treatment identified in the present study may provide a rationale to explore the optimal starting dose of lenvatinib.

In line with the high incidence of AEs associated with TKIs and the rarity of cure, the timing of TKI initiation is one of the major clinical issues. DTC and MTC generally grow slowly, and patients with metastatic disease may live without any symptoms for long periods. Therefore, the use of TKIs should be limited to the patients with progressive disease or with tumor-related symptoms such as diarrhea caused by MTC [59]. The doubling time of serum markers, thyroglobulin for DTC and calcitonin for MTC, has been investigated to predict disease progression $[60,61]$. These markers should be taken into consideration together with the radiographic assessment for the decision when to start TKIs.

Given the rarity of CR with TKI treatment identified in this meta-analysis, the importance of initial surgery as the only treatment to accomplish a cure may stand out. As for DTC, TKI treatment is currently restricted to patients with RR-DTC. Therefore, if complete resection can be accomplished in locally advanced DTC, surgery followed by RAI therapy should be the first choice. In DTC patients with distant metastases or high risk for future relapse, total thyroidectomy should be performed to remove all normal follicular thyroid cells that take iodine up before the initiation of RAI therapy. Therefore, surgery should preserve its important role in DTC treatment even in the era of TKIs. However, due to the limited number of hospitals providing RAI therapy in Japan, the waiting periods for RAI therapy are long. Another clinical concern is that metastatic disease can progress during the levothyroxine withdrawal before RAI therapy. Additionally, clinicians often encounter a patient with a distant metastasis from DTC who has previously been treated with hemithyroidectomy or subtotal thyroidectomy. Completion total thyroidectomy should be performed before RAI therapy, but a second surgery is associated with higher complication risks than the initial surgery, including the risk of permanent hypoparathyroidism [62]. Considering these unfavorable clinical concerns associated with RAI therapy, the implementation of a treatment strategy where a TKI is administrated without RAI therapy (RAI-skip therapy) would be one of the next clinical challenges that may increase the number of patients benefitting from TKIs.

To explore novel therapeutic strategies that further improve the treatment outcomes in the era of TKIs, several phase II/III studies are now ongoing (Table 6). Novel TKIs (anlotinib, apatinib, and donafenib) are currently tested in phase III clinical trials for thyroid cancer (NCT 02586337, NCT3048877, and NCT03602495). In addition to such single TKI therapies, phase II clinical trials are currently ongoing to evaluate the efficacy and safety of combined therapies including TKIs and immune checkpoint inhibitors (pembrolizumab, nivolmab, ipilimumab, and atezolizumab) (NCT02973997, NCT03914300, and NCT03170960). Besides TKI treatment, B-Raf kinase (BRAF) and MEK inhibitors (dabrafenib and trametinib) were recently approved by the FDA for ATC treatment based on the results of a phase II study [63]. For DTC, this combination therapy is currently being tested in phase II studies (NCT03244956 and NCT01723202), and improved outcomes are expected. As another therapeutic option, entrectinib, a potent inhibitor of the tropomyosin receptor kinase (TRK) [64], is currently available for patients with $N T R K$-fusion gene positive thyroid cancer in Japan.

As another potent therapeutic strategy, several studies indicate the feasibility of neoadjuvant molecular targeted therapies including TKIs and BRAF/MEK inhibitors followed by surgery. Milner et al. reported an advanced MTC case, in which vandetanib-induced local tumor shrinkage enabled the surgical resection of the primary site [65]. In addition, the results of case studies, which tested the efficacy of neoadjuvant dabrafenib plus trametinib followed by surgery in patients with $B R A F^{\mathrm{V} 600 \mathrm{E}}$ mutation-positive ATC, have recently been published 
Table 6 Currently recruiting and ongoing clinical trials (phase II or III) for thyroid cancer treatment

\begin{tabular}{lcccc}
\hline Drugs & Tumor & Study type & Status & NCT\# \\
\hline Anlotinib & DTC & Phase II/III & Active, not recruiting & NCT02586337 \\
Apatinib & DTC & Phase III & Recruiting & NCT03048877 \\
Donafenib & DTC & Phase III & Recruiting & NCT03602495 \\
Lenvatinib + Pembrolizumab & DTC & Phase II & Recruiting & NCT02973997 \\
Cabozantinib + Nivolmab + Ipilimumab & DTC & Phase II & Recruiting & NCT03914300 \\
Cabozantinib + Atezolizumab & MTC & Phase I/II & Recruiting & NCT03170960 \\
Dabrafenib + Trametinib & DTC & Phase II & Recruiting & NCT03244956 \\
Dabrafenib \pm Trametinib & DTC & Phase II & Active, not recruiting & NCT01723202 \\
\hline
\end{tabular}

DTC, differentiated thyroid carcinoma; MTC, medullary thyroid carcinoma

[66]. In this study, six $B R A F^{\mathrm{V} 600 \mathrm{E}}$ mutation positive ATC patients were enrolled, and a complete resection following neoadjuvant dabrafenib plus trametinib was achieved for all six patients. Furthermore, four patients were free of local, regional, and distant metastases over the course of one year. These results demonstrate the feasibility of surgery after neoadjuvant dabrafenib and trametinib therapy for $B R A F^{\mathrm{V} 600 \mathrm{E}}$ mutation-positive ATC. In addition, adjuvant external beam radiotherapy following surgery reportedly prolongs the survival of patients with ATC [6, 9, 67, 68]. Therefore, a multimodal treatment including neoadjuvant molecular targeted therapies, surgery, and external beam radiotherapy may be a potent therapeutic strategy for ATC. However, some clinical concerns may be associated with this strategy. One is that ATC may progress rapidly during the drug withdrawal periods before surgery, and the other is that the combined therapy may increase surgery-related AEs such as postoperative wound dehiscence. Therefore, further prospective studies are required to evaluate the benefits from this strategy.

Our study has several limitations. First, no clinical trials have compared each TKI directly for any pathological type of thyroid cancer. Second, a significant heterogeneity was observed in some meta-analyses. Third, this meta-analysis included retrospective studies. These limitations may impair the reliability of the results. Therefore, future RCTs, which carry out a oneon-one comparison of each TKI with a large number of patients, are needed to verify the findings of this metaanalysis.

In conclusion, the elucidation of the differences in the efficacy and frequently observed AEs of the currently available TKIs would provide a better understanding of thyroid cancer treatment. Future studies should focus on the exploration of the optimal TKI treatment choice for each patient, further improvement of patient outcomes through initial dose optimization, and development of novel therapeutic strategies including new TKIs, combination therapy of TKIs and other anti-cancer drugs such as ICIs, and other molecular targeted therapy including BRAF and MEK inhibitors. Even in the era of TKIs, a multimodal therapeutic approach is required for improved survival.

\section{Acknowledgement}

We would like to thank Editage (www.editage.com) for English language editing.

\section{Disclosure Statement}

The authors have no potential conflicts of interest associated with the research.

\section{References}

1. Hayat MJ, Howlader N, Reichman ME, Edwards BK (2007) Cancer statistics, trends, and multiple primary cancer analyses from the Surveillance, Epidemiology, and End Results (SEER) Program. Oncologist 12: 20-37.

2. Lim H, Devesa SS, Sosa JA, Check D, Kitahara CM (2017) Trends in thyroid cancer incidence and mortality in the United States, 1974-2013. JAMA 317: 1338-1348.
3. Ito Y, Higashiyama T, Takamura Y, Kobayashi K, Miya A, et al. (2010) Clinical outcomes of patients with papillary thyroid carcinoma after the detection of distant recurrence. World J Surg 34: 2333-2337.

4. Durante C, Haddy N, Baudin E, Leboulleux S, Hartl D, et al. (2006) Long-term outcome of 444 patients with distant metastases from papillary and follicular thyroid carci- 
noma: benefits and limits of radioiodine therapy. $J$ Clin Endocrinol Metab 91: 2892-2899.

5. Ito Y, Miyauchi A, Ito M, Yabuta $\mathrm{T}$, Masuoka $\mathrm{H}$, et al. (2014) Prognosis and prognostic factors of differentiated thyroid carcinoma after the appearance of metastasis refractory to radioactive iodine therapy. Endocr J 61: 821824.

6. Sugitani I, Miyauchi A, Sugino K, Okamoto T, Yoshida A, et al. (2012) Prognostic factors and treatment outcomes for anaplastic thyroid carcinoma: ATC Research Consortium of Japan cohort study of 677 patients. World J Surg 36: $1247-1254$.

7. Pierie JP, Muzikansky A, Gaz RD, Faquin WC, Ott MJ (2002) The effect of surgery and radiotherapy on outcome of anaplastic thyroid carcinoma. Ann Surg Oncol 9: 5764.

8. Ito Y, Higashiyama T, Hirokawa M, Fukushima M, Inoue $\mathrm{H}$, et al. (2009) Investigation of the validity of UICC stage grouping of anaplastic carcinoma of the thyroid. Asian $J$ Surg 32: 47-50.

9. Ito K, Hanamura T, Murayama K, Okada T, Watanabe T, et al. (2012) Multimodality therapeutic outcomes in anaplastic thyroid carcinoma: improved survival in subgroups of patients with localized primary tumors. Head Neck 34: 230-237.

10. Are C, Shaha AR (2006) Anaplastic thyroid carcinoma: biology, pathogenesis, prognostic factors, and treatment approaches. Ann Surg Oncol 13: 453-464.

11. Ito Y, Onoda N, Okamoto T (2020) The revised clinical practice guidelines on the management of thyroid tumors by the Japan Associations of Endocrine Surgeons: core questions and recommendations for treatments of thyroid cancer. Endocr J 67: 669-717.

12. Wells SA Jr, Asa SL, Dralle H, Elisei R, Evans DB, et al. (2015) Revised American Thyroid Association guidelines for the management of medullary thyroid carcinoma. Thyroid 25: 567-610.

13. Ito $\mathrm{Y}$, Miyauchi $\mathrm{A}$, Kihara $\mathrm{M}$, Higashiiyama $\mathrm{T}$, Fukushima M, et al. (2018) Static prognostic factors and appropriate surgical designs for patients with medullary thyroid carcinoma: the second report from a singleinstitution study in Japan. World J Surg 42: 3954-3966.

14. Schlumberger M, Tahara M, Wirth LJ, Robinson B, Brose MS, et al. (2015) Lenvatinib versus placebo in radioiodine-refractory thyroid cancer. $N$ Engl J Med 372: 621-630.

15. Brose MS, Nutting CM, Jarzab B, Elisei R, Siena S, et al. (2014) Sorafenib in radioactive iodine-refractory, locally advanced or metastatic differentiated thyroid cancer: a randomised, double-blind, phase 3 trial. Lancet 384: 319 328.

16. Schlumberger M, Elisei R, Muller S, Schoffski P, Brose M, et al. (2017) Overall survival analysis of EXAM, a phase III trial of cabozantinib in patients with radiographically progressive medullary thyroid carcinoma. Ann Oncol 28: 2813-2819.

17. Wells SA Jr, Robinson BG, Gagel RF, Dralle H, Fagin JA, et al. (2012) Vandetanib in patients with locally advanced or metastatic medullary thyroid cancer: a randomized, double-blind phase III trial. J Clin Oncol 30: 134-141.

18. Ahmed M, Barbachano Y, Riddell A, Hickey J, Newbold KL, et al. (2011) Analysis of the efficacy and toxicity of sorafenib in thyroid cancer: a phase II study in a UK based population. Eur J Endocrinol 165: 315-322.

19. Gupta-Abramson V, Troxel AB, Nellore A, Puttaswamy $\mathrm{K}$, Redlinger M, et al. (2008) Phase II trial of sorafenib in advanced thyroid cancer. J Clin Oncol 26: 4714-4719.

20. Ito Y, Onoda N, Ito KI, Sugitani I, Takahashi S, et al. (2017) Sorafenib in Japanese patients with locally advanced or metastatic medullary thyroid carcinoma and anaplastic thyroid carcinoma. Thyroid 27: 1142-1148.

21. Lam ET, Ringel MD, Kloos RT, Prior TW, Knopp MV, et al. (2010) Phase II clinical trial of sorafenib in metastatic medullary thyroid cancer. J Clin Oncol 28: 2323-2330.

22. Schlumberger M, Jarzab B, Cabanillas ME, Robinson B, Pacini F, et al. (2016) A phase II trial of the multitargeted tyrosine kinase inhibitor lenvatinib (E7080) in advanced medullary thyroid cancer. Clin Cancer Res 22: 44-53.

23. Takahashi S, Kiyota N, Yamazaki T, Chayahara N, Nakano K, et al. (2019) A phase II study of the safety and efficacy of lenvatinib in patients with advanced thyroid cancer. Future Oncol 15: 717-726.

24. Borenstein M, Hedges LV, Higgins JP, Rothstein HR (2010) A basic introduction to fixed-effect and randomeffects models for meta-analysis. Res Synth Methods 1: 97-111.

25. Kloos RT, Ringel MD, Knopp MV, Hall NC, King M, et al. (2009) Phase II trial of sorafenib in metastatic thyroid cancer. J Clin Oncol 27: 1675-1684.

26. Cabanillas ME, Waguespack SG, Bronstein Y, Williams MD, Feng L, et al. (2010) Treatment with tyrosine kinase inhibitors for patients with differentiated thyroid cancer: the M. D. Anderson experience. J Clin Endocrinol Metab 95: 2588-2595.

27. Frank-Raue K, Ganten M, Kreissl MC, Raue F (2011) Rapid response to sorafenib in metastatic medullary thyroid carcinoma. Exp Clin Endocrinol Diabetes 119: 151155.

28. Capdevila J, Iglesias L, Halperin I, Segura A, MartinezTrufero J, et al. (2012) Sorafenib in metastatic thyroid cancer. Endocr Relat Cancer 19: 209-216.

29. Schneider TC, Abdulrahman RM, Corssmit EP, Morreau H, Smit JW, et al. (2012) Long-term analysis of the efficacy and tolerability of sorafenib in advanced radio-iodine refractory differentiated thyroid carcinoma: final results of a phase II trial. Eur J Endocrinol 167: 643-650.

30. Savvides P, Nagaiah G, Lavertu P, Fu P, Wright JJ, et al. (2013) Phase II trial of sorafenib in patients with advanced anaplastic carcinoma of the thyroid. Thyroid 23: 600-604.

31. Benekli M, Yalcin S, Ozkan M, Elkiran ET, Sevinc A, et al. (2015) Efficacy of sorafenib in advanced differentiated and medullary thyroid cancer: experience in a Turkish population. Onco Targets Ther 8: 1-5.

32. de Castroneves LA, Negrao MV, de Freitas RM, Papadia 
C, Lima JV Jr, et al. (2016) Sorafenib for the treatment of progressive metastatic medullary thyroid cancer: efficacy and safety analysis. Thyroid 26: 414-419.

33. Kocsis J, Szekanecz E, Bassam A, Uhlyarik A, Papai Z, et al. (2019) First line sorafenib treatment for metastatic medullary thyroid cancer: efficacy and safety analysis. Exp Clin Endocrinol Diabetes 127: 240-246.

34. Balmelli C, Railic N, Siano M, Feuerlein K, Cathomas R, et al. (2018) Lenvatinib in advanced radioiodinerefractory thyroid cancer-a retrospective analysis of the Swiss Lenvatinib Named Patient Program. J Cancer 9: 250-255.

35. Cabanillas ME, Schlumberger M, Jarzab B, Martins RG, Pacini F, et al. (2015) A phase 2 trial of lenvatinib (E7080) in advanced, progressive, radioiodine-refractory, differentiated thyroid cancer: a clinical outcomes and biomarker assessment. Cancer 121: 2749-2756.

36. Iniguez-Ariza NM, Ryder MM, Hilger CR, Bible KC (2017) Salvage lenvatinib therapy in metastatic anaplastic thyroid cancer. Thyroid 27: 923-927.

37. Iwasaki H, Yamazaki H, Takasaki H, Suganuma N, Nakayama H, et al. (2018) Lenvatinib as a novel treatment for anaplastic thyroid cancer: a retrospective study. Oncol Lett 16: 7271-7277.

38. Iyer PC, Dadu R, Ferrarotto R, Busaidy NL, Habra MA, et al. (2018) Real-world experience with targeted therapy for the treatment of anaplastic thyroid carcinoma. Thyroid 28: $79-87$.

39. Koyama S, Miyake N, Fujiwara K, Morisaki T, Fukuhara $\mathrm{T}$, et al. (2018) Lenvatinib for anaplastic thyroid cancer and lenvatinib-induced thyroid dysfunction. Eur Thyroid $J$ 7: 139-144.

40. Nervo A, Gallo M, Sama MT, Felicetti F, Alfano M, et al. (2018) Lenvatinib in advanced radioiodine-refractory thyroid cancer: a snapshot of real-life clinical practice. Anticancer Res 38: 1643-1649.

41. Staub Y, Nishiyama A, Suga Y, Fujita M, Matsushita R, et al. (2019) Clinical characteristics associated with lenvatinib-induced fistula and tumor-related bleeding in patients with thyroid cancer. Anticancer Res 39: 38713878.

42. Suzuki C, Kiyota N, Imamura Y, Goto H, Suto H, et al. (2019) Exploratory analysis of prognostic factors for lenvatinib in radioiodine-refractory differentiated thyroid cancer. Head Neck 41: 3023-3032.

43. Yamazaki H, Iwasaki H, Takasaki H, Suganuma N, Sakai $\mathrm{R}$, et al. (2019) Efficacy and tolerability of initial lowdose lenvatinib to treat differentiated thyroid cancer. Medicine (Baltimore) 98: e14774.

44. Wells SA Jr, Gosnell JE, Gagel RF, Moley J, Pfister D, et al. (2010) Vandetanib for the treatment of patients with locally advanced or metastatic hereditary medullary thyroid cancer. J Clin Oncol 28: 767-772.

45. Leboulleux S, Bastholt L, Krause T, de la Fouchardiere C, Tennvall J, et al. (2012) Vandetanib in locally advanced or metastatic differentiated thyroid cancer: a randomised, double-blind, phase 2 trial. Lancet Oncol 13: 897-905.
46. Werner RA, Schmid JS, Higuchi T, Javadi MS, Rowe SP, et al. (2018) Predictive value of (18)F-FDG PET in patients with advanced medullary thyroid carcinoma treated with vandetanib. J Nucl Med 59: 756-761.

47. Hu MI, Elisei R, Dedecjus M, Popovtzer A, Druce M, et al. (2019) Safety and efficacy of two starting doses of vandetanib in advanced medullary thyroid cancer. Endocr Relat Cancer 26: 241-250.

48. Elisei R, Schlumberger MJ, Muller SP, Schoffski P, Brose MS, et al. (2013) Cabozantinib in progressive medullary thyroid cancer. J Clin Oncol 31: 3639-3646.

49. Cabanillas ME, de Souza JA, Geyer S, Wirth LJ, Menefee ME, et al. (2017) Cabozantinib as salvage therapy for patients with tyrosine kinase inhibitor-refractory differentiated thyroid cancer: results of a multicenter phase II International Thyroid Oncology Group trial. J Clin Oncol 35: 3315-3321.

50. Je Y, Schutz FA, Choueiri TK (2009) Risk of bleeding with vascular endothelial growth factor receptor tyrosinekinase inhibitors sunitinib and sorafenib: a systematic review and meta-analysis of clinical trials. Lancet Oncol 10: $967-974$.

51. Cavalieri S, Cosmai L, Genderini A, Nebuloni M, Tosoni A, et al. (2018) Lenvatinib-induced renal failure: two firsttime case reports and review of literature. Expert Opin Drug Metab Toxicol 14: 379-385.

52. Hyogo Y, Kiyota N, Otsuki N, Goto S, Imamura Y, et al. (2018) Thrombotic microangiopathy with severe proteinuria induced by lenvatinib for radioactive iodine-refractory papillary thyroid carcinoma. Case Rep Oncol 11: 735741.

53. Pandey AK, Singhi EK, Arroyo JP, Ikizler TA, Gould ER, et al. (2018) Mechanisms of VEGF (Vascular Endothelial Growth Factor) Inhibitor-associated hypertension and vascular disease. Hypertension 71: e1-e8.

54. Tassi E, Lai EY, Li L, Solis G, Chen Y, et al. (2018) Blood pressure control by a secreted FGFBP1 (Fibroblast Growth Factor-Binding Protein). Hypertension 71: 160167.

55. Mizukami Y, Sugawara K, Kira Y, Tsuruta D (2017) Sorafenib stimulates human skin type mast cell degranulation and maturation. J Dermatol Sci 88: 308-319.

56. Brose MS, Frenette CT, Keefe SM, Stein SM (2014) Management of sorafenib-related adverse events: a clinician's perspective. Semin Oncol 41 Suppl 2: S1-S16.

57. Cabanillas ME, Takahashi S (2019) Managing the adverse events associated with lenvatinib therapy in radioiodinerefractory differentiated thyroid cancer. Semin Oncol 46: $57-64$.

58. Tahara M, Brose MS, Wirth LJ, Suzuki T, Miyagishi H, et al. (2019) Impact of dose interruption on the efficacy of lenvatinib in a phase 3 study in patients with radioiodinerefractory differentiated thyroid cancer. Eur J Cancer 106: 61-68.

59. Pais MC, Maia T, Peixoto A, Meira L, Albuquerque A, et al. (2018) Diarrhea as a form of presentation of medullary thyroid carcinoma. Porto Biomed J 3: e18. 
60. Laure Giraudet A, Al Ghulzan A, Auperin A, Leboulleux S, Chehboun A, et al. (2008) Progression of medullary thyroid carcinoma: assessment with calcitonin and carcinoembryonic antigen doubling times. Eur J Endocrinol 158: 239-246.

61. Miyauchi A, Kudo T, Miya A, Kobayashi K, Ito Y, et al. (2011) Prognostic impact of serum thyroglobulin doubling-time under thyrotropin suppression in patients with papillary thyroid carcinoma who underwent total thyroidectomy. Thyroid 21: 707-716.

62. Ito Y, Kihara M, Kobayashi K, Miya A, Miyauchi A (2014) Permanent hypoparathyroidism after completion total thyroidectomy as a second surgery: How do we avoid it? Endocr J 61: 403-408.

63. Subbiah V, Kreitman RJ, Wainberg ZA, Cho JY, Schellens JHM, et al. (2018) Dabrafenib and trametinib treatment in patients with locally advanced or metastatic BRAF V600-mutant anaplastic thyroid cancer. J Clin Oncol 36: 7-13.

64. Doebele RC, Drilon A, Paz-Ares L, Siena S, Shaw AT, et al. (2020) Entrectinib in patients with advanced or meta- static NTRK fusion-positive solid tumours: integrated analysis of three phase 1-2 trials. Lancet Oncol 21: 271282.

65. Milner TD, Ronghe M, Shaikh MG, MacGregor FB, Reed $\mathrm{N}$ (2019) Vandetanib tumor shrinkage in metastatic medullary thyroid cancer allowing surgical resection of the primary site: a case report. J Pediatr Hematol Oncol 41: e329-e332.

66. Wang JR, Zafereo ME, Dadu R, Ferrarotto R, Busaidy NL, et al. (2019) Complete surgical resection following neoadjuvant dabrafenib plus trametinib in BRAF(V600E)mutated anaplastic thyroid carcinoma. Thyroid 29: 10361043.

67. Saeed NA, Kelly JR, Deshpande HA, Bhatia AK, Burtness BA, et al. (2020) Adjuvant external beam radiotherapy for surgically resected, nonmetastatic anaplastic thyroid cancer. Head Neck 42: 1031-1044.

68. Takahashi N, Matsushita H, Umezawa R, Yamamoto T, Ishikawa Y, et al. (2019) Hypofractionated radiotherapy for anaplastic thyroid carcinoma: 15 years of experience in a single institution. Eur Thyroid J 8: 24-30. 